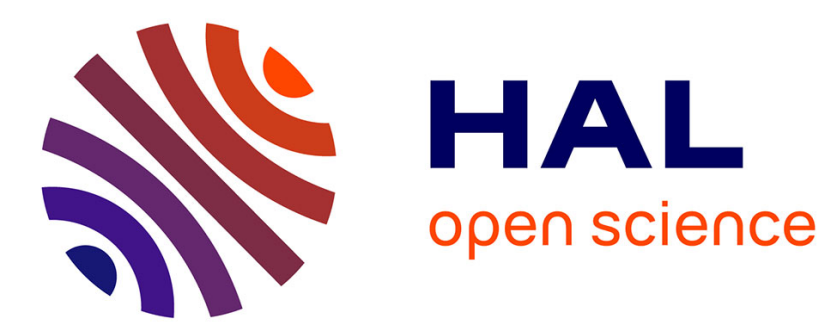

\title{
The inducer maltotriose binds in the central cavity of the tetratricopeptide-like sensor domain of MalT, a bacterial STAND transcription factor
}

Olivier Danot

\section{- To cite this version:}

Olivier Danot. The inducer maltotriose binds in the central cavity of the tetratricopeptide-like sensor domain of MalT, a bacterial STAND transcription factor. Molecular Microbiology, 2010, 77 (3), pp.628. 10.1111/j.1365-2958.2010.07237.x . hal-00552641

\section{HAL Id: hal-00552641 \\ https://hal.science/hal-00552641}

Submitted on 6 Jan 2011

HAL is a multi-disciplinary open access archive for the deposit and dissemination of scientific research documents, whether they are published or not. The documents may come from teaching and research institutions in France or abroad, or from public or private research centers.
L'archive ouverte pluridisciplinaire HAL, est destinée au dépôt et à la diffusion de documents scientifiques de niveau recherche, publiés ou non, émanant des établissements d'enseignement et de recherche français ou étrangers, des laboratoires publics ou privés. 


\section{molecular microbiology}

The inducer maltotriose binds in the central cavity of the tetratricopeptide-like sensor domain of MalT, a bacterial STAND transcription factor

\begin{tabular}{|r|l|}
\hline Journal: & Molecular Microbiology \\
\hline Manuscript ID: & MMI-2010-10013.R1 \\
\hline Manuscript Type: & Research Article \\
\hline Date Submitted by the \\
Author: & $19-$ May-2010 \\
\hline Complete List of Authors: & Danot, Olivier; Institut Pasteur, Microbiology Department \\
\hline Key Words: & $\begin{array}{l}\text { MalT, STAND ATPase, ligand binding, tetratricopeptide repeat, } \\
\text { maltotriose }\end{array}$ \\
\hline
\end{tabular}

\section{ScholaronE}

Manuscript Central 


\section{The inducer maltotriose binds in the central cavity of the}

\section{2 tetratricopeptide-like sensor domain of MalT, a bacterial STAND}

\section{3 transcription factor}

4

5 Olivier Danot ${ }^{*}$

6

Institut Pasteur, Molecular Genetics Unit and CNRS URA 2172, 25, rue du Dr Roux, 75724 Paris cedex, France

9

Running title: Maltotriose-binding site of MalT sensor domain

\# Abbreviations: STAND, Signal Transduction ATPases with Numerous Domains; NOD, nucleotide

21 Oligomerization Domain; SUPR, Superhelical Repeats; AAA+ ATPases, ATPases associated with Pyrin domain containing; TPR, tetratricopeptide repeats; DT3, sensor domain of MalT; rmsd, root mean square deviation; Ni-NTA, nickel-nitrilotriacetic acid. 


\section{Summary}

2 Signal transduction ATPases with numerous domains (STAND) are sophisticated proteins that 3 integrate several signals and respond by building multimeric platforms allowing signaling in various

4 processes: apoptosis, innate immunity, bacterial metabolism. They comprise a conserved nucleotide 5 oligomerization domain (NOD), which functions as a binary switch that oscillates between the OFF 6 (ADP-bound) and the ON (ATP-bound) conformation, and non conserved sensor and effector 7 domains. Transition from the OFF form to the ON form strictly depends on the binding of an inducer to 8 the sensor domain. The interaction of the inducer with this domain was studied in MalT, a model 9 STAND protein. MalT sensor domain has a SUPR (superhelical repeats) fold resembling a cylinder 10 with a central cavity. The cavity was subjected to an alanine-scanning approach, and the effects of the 11 alanine substitutions on inducer binding and transcription activation were analyzed. This work 12 unambiguously showed that the inducer maltotriose binds inside the cavity, and a patch on the inner 13 surface was proposed to be the primary maltotriose binding-site. Furthermore, limited proteolysis 14 suggested that maltotriose binding changes the conformation of the sensor domain. 


\section{Introduction}

AAA+ ATPases constitute a large class of P-loop ATPases functioning as multimers and involved in extremely diverse cellular activities. Signal Transduction ATPases with Numerous Domains (STAND) are atypical AAA+ (Erzberger et al., 2006) involved in signal transduction (Danot et al., 2009; Leipe et al., 2004) that are able to assemble into large multimeric scaffolds upon integrating several positive and negative signals. These platforms bring together target molecules of various kinds or carry out enzymatic reactions, which triggers downstream signaling. Signaling by STAND proteins pertains to various cellular functions: for example apoptosis (like the proapoptotic factor APAF1), innate and adaptive immunity (the numerous NLRP and plant R proteins, NOD1 and NOD2, CIITA) in eukaryotes. In prokaryotes, STAND have also multiple roles: primary or secondary metabolism, like transcription activators MalT (regulator of the Escherichia coli maltose regulon, Boos and Shuman, 1998) and AfsR (a global regulator controlling antibiotic production in Streptomyces cœlicolor, Tanaka et al., 2007) but also envelope integrity and probably survival in activated macrophages, like Mycobacterium tuberculosis serine/threonine kinase PknK (Kumar et al., 2009 and references therein).

STAND proteins share a multidomain architecture (Fig. 1A) comprising a NOD (Nucleotidebinding Oligomerization Domain) conserved module, followed by a non-conserved sensor domain sometimes separated from it by a short spacer called the arm. This architecture is completed by one or several effector domains, which lie at either end of the NOD-sensor polypeptide. The NOD module is the centerpiece of the mechanism of action of STAND proteins. The NOD consists of the conserved core of AAA+ ATPases, with Walker boxes $A$ and $B$, followed by a winged helix domain specific to the STAND. The presence of this domain enables the NOD module to act as a binary switch that oscillates between two conformations: an ADP-bound long-lived OFF form (inactive) and an ATP-bound ON form prone to multimerize (Bao et al., 2007; Marquenet and Richet, 2007; Riedl et al., 2005; Tameling et al., 2006), which leads to the multimeric platform that is the active species.

Signaling by STAND proteins relies on the control of this NOD binary switch by an inducer, i.e. a molecule that binds and activates the protein (Bao et al., 2007; Marquenet and Richet, 2007; Schreiber and Richet, 1999). However, no direct interaction between the inducer and the NOD was observed in any STAND protein, whereas at least in two cases, those of the rice R protein Pi-ta (Jia et al., 2000) and the MalT transcription activator (Danot, 2001), the inducer bound the sensor domain, hence its name. Yet, the precise mechanism of action of the inducer on the NOD switch is unknown. Deletion of the sensor domain in STAND proteins had opposite results depending on the protein. In certain 
1 instances it caused protein activation in the absence of inducer (Ade et al., 2007; Hsu et al., 2008; Hu et al., 1998; Tanabe et al., 2004), leading to the notion that the apo-sensor domain maintains the NOD in the OFF position till inducer binding to the sensor relieves this inhibition. In other cases deletion of the sensor abolished the activity, which could sometimes be restored by co-producing the sensor domain in trans (Moffett et al., 2002), suggesting that this domain can also play a positive role.

Sensor domains are not conserved throughout the STAND family. They belong almost exclusively to four classes of superstructure-forming domains made of repeated motifs: leucine-rich repeats, WD40 repeats, tetratricopeptide repeats (TPR), and superhelical repeats (SUPR), a variation of the TPR. Bacterial proteins of the STAND family have a sensor domain either of the TPR or of the SUPR kind, the latter being specific of the MalT/PknK clade. Even though numerous WD40, leucine-rich repeats and TPR domains have been crystallized in other contexts, the only STAND sensor domain whose three-dimensional structure is known to date is that of MalT (abbreviated as DT3, Steegborn et al., 2001). Like TPR, SUPR are made of a two $\alpha$-helix antiparallel bundle motif, but SUPR helices are longer and the motif is more degenerated. In both cases, the succession of the $\alpha$-helices forms a righthanded solenoid with adjacent bundles parallelly stacking against each other (Fig. 1B, inset), whose envelope has the shape of a superhelical slab. In the MaIT SUPR structure the superhelical slab winds upon itself to form a hollow cylinder, due to the head-to-tail stacking of helix bundles one superhelical turn (i.e. 5 bundles) apart. The cylinder is closed at one end by a C-terminal three-helix subdomain. It has an axial opening (Fig. 1C, left panel) at the other end of the cylinder (near the $\mathrm{N}$-terminus) and a second, lateral opening between the contact point between bundles 1 and 6 and that between bundles 2 and 7 (Fig. 1C, right panel).

Whereas inducer binding is a key event in STAND signaling, no inducer binding site of a STAND protein has been characterized yet. To study the molecular basis of inducer-sensor domain interaction on the model of the MalT transcription activator, an alanine-scanning mutagenesis approach was undertaken. Functional analyses revealed the precise location of the binding site for the inducer maltotriose and suggests that this binding changes the conformation of the sensor domain.

\section{Results}

Strategy

Statistics bearing on ligand-binding proteins with a known three-dimensional structure show that the ligand binding site is harbored by the largest cavity on the surface of the protein in about $70 \%$ of 
1 the cases (Nayal and Honig, 2006). A candidate for inducer binding in the MalT sensor domain is 2 therefore the large central cavity (3530 $\AA^{3}$ as calculated using the CASTp software (molecular surface 3 calculation (Dundas et al., 2006)). The Van der Waals volume of a maltotriose molecule is only $420 \AA^{3}$ 4 (calculated as described (Zhao et al., 2003)). There are therefore numerous ways in which maltotriose could be accommodated in this cavity. To determine whether maltotriose binds in this pocket, and to accurately locate its binding site, residues that line the cavity (identified by CASTp (Table S2)) were individually replaced by alanines. Glycine and proline residues were excluded from this analysis. Since MalT proteins from other bacteria are expected to bind the same inducer (maltotriose) in the same way as E. coli MalT, the analysis was also restricted to residues conserved in a set of 6 MalT sequences* . According to these rules, 29 codons (Fig. 1B) of the part of the malT gene encoding DT3 borne by an M13 vector were individually changed to alanine codons by site-directed mutagenesis, thus creating a homogeneous library of sensor domain mutations.

\section{A majority of the mutations affect MalT activity in vivo}

The pop8083 strain bears a malP'-'lacZYA fusion at the malPQ locus, which directs the synthesis of a hybrid MalP'-'LacZ active $\beta$-galactosidase under the control of the malPp MalT-dependent 17 promoter. The malK-malM region of this strain is replaced by a zeocin resistance cassette: in the absence of the MalK protein, which is known to inhibit MalT activity, MalT-dependent transcription becomes constitutive, and this constitutivity is at least partially due to the presence of endogenous maltotriose in the cytoplasm. All the mutations were transferred to the chromosomal malT gene of

21 pop8083, strains were grown at $25^{\circ} \mathrm{C}$ and their $\beta$-galactosidase activity was assayed.

* The MalT sequences used were those from gammaproteobacteria Aggregatibacter actinomycetemcomitans, Actinobacillus pleuropneumoniae, Photorhabdus luminescens, Vibrio cholerae, Vibrio vulnificus, Yersinia pseudotuberculosis. They are all annotated as MalT proteins by the NCBI (http://www.ncbi.nlm.nih.gov/) and the corresponding genes map immediately next to orthologs of malP and malQ, as in the E. coli chromosome. In addition, the proteins of $V$. cholerae and $V$. vulnificus (those with the lowest percentage of identity with E. coli MalT) have been purified and showed maltotriose dependent multimerization like E. coli MalT (Danot, unpublished results). 
A majority of the mutations had a deleterious effect on MalT activity (Fig. 2), with those belonging to the $\mathrm{N}$-terminal part of the domain (i.e. close to the openings of the cavity) being in general more detrimental. Only 5 mutations out of 29 did not decrease the activity of the malPp promoter. Among these mutations, D647A, Q690A and N693A did not alter MalT activity, while R729A and R692A increased it. Three substitutions (W581A, R696A and 1769A) caused complete abolition of MalT activity. A western blot of soluble extracts prepared from cultures of all the pop8083 derivatives grown in the conditions of the $\beta$-galactosidase assays (Fig. 2, bottom panel) shows that the amount of soluble MalT was not significantly affected by the mutations: after quantification, all strains appeared to produce the MalT variants at a level within $20 \%$ (close to the experimental error) of the wt except for W529A (70\%), D647A (150\%), W648A (125\%), R696A (55\%), I769A (60\%). In conclusion, a majority of the residues that line the large central cavity of the MalT sensor domain and that are conserved in MalT orthologs seem to play a role in the activity of MalT. These observations are compatible with a role of these residues in the binding of maltotriose and suggest that the inducer binding-site is indeed contained by the large central cavity of the sensor domain.

Purification of the variants of the isolated sensor domain.

The $\beta$-galactosidase assays described above evaluate the influence of the substituted residues on the global signal transduction process carried out by MalT. To determine which residues of the sensor domain play a role in the physical interaction with maltotriose, the mutations were transferred in the context of the isolated sensor domain. The mutations were introduced in the gene encoding DT3 present in a pET28b(+) derived expression plasmid, pOM164, harbored by the BL21(DE3) $\Delta$ malT220 strain. In this expression system, wild-type DT3 and all variants could be produced in a soluble form except W581A, R692A, R696A and 1769A. Proteins were purified by a one-step Ni-NTA chromatography. R692A, but not the three other insoluble variants, could be rescued by replacing IPTG induction by autoinduction (Studier, 2005). Moreover, to obtain R692A at sufficient concentration and purity, it was purified as a $\mathrm{His}_{6}$-tagged version named hR692A, along with a control $\mathrm{His}_{6}$-tagged version of DT3, hDT3. Finally, a total of 26 variants of the MalT sensor domain could be purified.

Noteworthy, the three mutations W581A, R696A and I769A that completely abolish $\beta$ - 
1 suggests that the effect of these mutations is due to a global destabilization of the structure. Note that

$2 R 696 \mathrm{~A}$ and I769A decrease the amount of soluble MalT in vivo even at low protein levels, as shown by 3 the Western blot on Fig. 2, and that W581 is the most buried tryptophan residue of the whole domain.

4 Interestingly, these mutations all affect residues involved in the interaction between the SUPR repeats 5 and the three helix C-terminal subdomain: W581 (bundle 4) interacts with S765 (C-terminal subdomain), 1769 (C-terminal subdomain) with M662 (bundle 6), and R696 (bundle 7) with E770 (Cterminal subdomain). Interactions between the SUPR fold and the C-terminal subdomain, which could play the role of the solvation or capping helix in TPR structures (Main et al., 2003), might therefore be important for the overall stability of the sensor domain.

Effect of the mutations on maltotriose affinity: a fluorescence study

The MalT sensor domain comprises 11 tryptophan residues, which means that its fluorescence emission spectrum is highly composite. It has been observed that maltotriose binding exalts the overall intrinsic fluorescence of DT3 and shifts the maximum of the emission spectrum towards shorter wavelengths (Danot, 2001). To examine the effects of the substitutions on maltotriose binding to the sensor domain, fluorescence spectra of all purified variants were recorded in the presence and absence of maltotriose (Fig. 3A). In general, the shape of the spectra of the purified variants without maltotriose is nearly identical to that of the wt protein, suggesting that the 26 substitutions tested do not change the protein three-dimensional structure significantly. Only two mutations, D726A and W648A, had a significant effect on the global position of the spectrum without maltotriose: they shifted it towards short wavelengths (Table 1). A straightforward explanation in the case of W648A is that W648 has a maximum fluorescence emission in the vicinity of $350 \mathrm{~nm}$ (consistent with the fact that it is exposed to the solvent) and therefore contributes more to the right part of the overall fluorescence spectrum.

The maltotriose-induced changes in the fluorescence emission spectrum were differentially affected by the substitutions (Fig. 3A, Table 4). Out of 26 substitutions, 10 (group 1: E496A, H499A, W529A, Q533A, E536A, R574A, H645A, N684A, F686A, D726A) completely abolished the maltotriose-induced fluorescence increase, suggesting that the corresponding residues play an important role in maltotriose binding. Spectra of other variants like N693A or Q690A responded to maltotriose in the same way as the wt, whereas others had intermediate spectral changes. To quantify the differences between the variants, maltotriose binding curves were obtained by recording the 
1 Fig. $3 \mathrm{C}$ for examples of binding curves and Fig. S2 for the totality of the binding curves). Dissociation constants derived from these experiments are reported (Table 2). 3 substitutions decreased the affinity of the sensor domain for maltotriose by a factor of more than 10 (group 2: L504A, Q688A, L723A), 2 had a 5- or 6-fold effect (group 3: Q457A and H685A), and the 11 others (group 4: N461A, I537A, E570A, F571A, L600A, D647A, W648A, Q690A, hR692A, N693A, R729A) had less than a 3-fold effect. The large number of residues of the cavity whose substitution impacts maltotriose binding corroborates the in vivo study and strongly suggests that the cavity of the MalT sensor domain contains the maltotriose-binding site. To be more specific, residues of group 1 are most probably involved in maltotriose binding, while residues of group 2 and 3 might play accessory roles, and residues of group 4 do not participate importantly in the binding of maltotriose to DT3.

\section{The primary maltotriose-binding site}

Fig. 4A shows a surface representation of DT3 split in two halves to show the wall of the cavity. All of the 26 altered residues are colored according to the group which they belong to. The right half contains fewer residues that are conserved in MalT orthologs (and that were therefore tested). All residues of group 1 and 2 but $\mathrm{H} 645$ and R574 form a contiguous patch. This patch extends between the two openings of the cavity but does not reach towards the bottom of the pocket. It is entirely contained in the left moiety of the cavity (as defined from Fig. 4A) and more precisely in a quadrilater whose summits are F686, E496, W529 and D726. It is contributed for by two pairs of helix bundles that are one superhelical turn apart: bundles 2 and 7, bundles 3 and 8 . The largest distance between any two aminoacids of this patch, $16.5 \AA$ (between D726 and W529) is slightly superior to the length of a maltotriose molecule $(\sim 13 \AA)$.

In order to determine how maltotriose could interact with the putative binding-site built up by group 1 residues, automated docking of maltotriose to the wall of the cavity was performed using AutoDock 4.0 (Goodsell and Olson, 1990; Morris et al., 1996; Morris et al., 1998). The 450 docked conformations obtained were grouped in clusters (sets of similar conformations) according to their mutual rmsd values with a $3.0 \AA$ tolerance. A large number of clusters was found (27 containing more than 4 conformations), possibly reflecting the relatively high number of torsions of the ligand and the large protein surface tested. The lowest energy cluster (Table 3 ) had only 3 members and the 4 largest clusters fell within $2.5 \mathrm{kcal} / \mathrm{mol}$ (the standard error of the force field (Huey et al., 2007)) of the lowest energy. Since the real conformation of the ligand can be approximated by the lowest energy conformation but also by a low-energy conformation from one of the most populated clusters (Bikadi 
1 and Hazai, 2009), maltotriose conformations within $2.5 \mathrm{kcal} / \mathrm{mol}$ from the lowest energy and belonging 2 to the 5 clusters mentioned above were studied further. To determine which one of these 3 conformations is most likely to correspond to the real position of the maltotriose molecule bound to 4 DT3, their average distance to group 1 residues was measured as described in Material and Methods. 5 Conformations of cluster 7 scored best according to this test. The best scoring conformation of cluster 67 (average distance to group 1 residues : $3.3 \AA$ ) is shown in Fig. 4A an 4C. The docked molecule, which lies roughly parallel to helix 3 , with its reducing end directed towards the bottom of the pocket, is closer than $3.7 \AA$ from 7 group 1 residues: E496, H499, W529, Q533, E536, F686 and D726, and several protein-ligand hydrogen bonds might be formed in this conformation (Fig. 4C). Moreover, it displays a good surface complementarity with the inner surface of the cavity (see Fig. 4A) and the central glucosyl unit is in a position to stack against F686, although imperfectly. For all these reasons, this theoretical conformation seems to be a reasonable working model of the bound maltotriose molecule. The 7 group 1 residues mentioned above belong to the contiguous patch described at the beginning of this chapter, which will be temptatively called the primary maltotriose binding site hereinafter.

Yet, H645, N684 and R574 (group 1) and Q457, H685, L504, L723 (groups 2 and 3) are more distant from the docked maltotriose. Three of them might play an indirect role by positioning important residues: N684 and H685 lie $\sim 3 \AA$ away from F686, and Q457 is hydrogen bonded to E496. As for L504 (bundle 2), L723 (turn between bundle 7 and 8, facing L504) and H645, they are probably important for the head-to-tail stacking of bundles 2 and 7 (H645A renders the R519-Q520 peptide bond sensitive to trypsin (not shown), suggesting a "breathing" of bundles 1 and 2 with respect to the rest of the domain).

Interaction of the last group 1 residue, R574, with maltotriose in the conformation of Fig.4C requires a long distance conformational change which is supported by the following observation. W648A, D648A and E570 variants have reduced maltotriose-induced blue-shifts (Table 1): the W648A substitution grossly mimics the absence of maltotriose, while D647A and E570A mimic its presence. An appealing hypothesis is that in the wt, maltotriose binding causes the two charged residues D647 and E570 to come closer to W648 and quench its fluorescence, which requires a long distance conformational change.

Maltotriose probably causes a global conformational change in the MalT sensor domain 
1 sensor domain, limited proteolysis of DT3 by trypsin was performed in the presence and absence of maltotriose. The presence of $200 \mathrm{mM}$ maltotriose drastically protected the domain from trypsin attack

3 (Fig. 5). Control experiments showed that protease sensitivity of a control protein was barely affected by $200 \mathrm{mM}$ maltotriose, as was trypsin sensitivity of DT3 by $200 \mathrm{mM}$ glucose. In the crystal used to solve the structure of the MalT sensor domain, the primary maltotriose binding site proposed here is not accessible to the trypsin catalytic site because it is contained by the central cavity. Therefore, maltotriose binding protects the sensor domain from trypsin proteolysis, not by directly shielding peptide bonds of this binding site, but rather by changing the conformation of more exposed peptide bonds from a distance. This strongly suggests that maltotriose elicits a long-range conformational change of DT3.

\section{Discussion}

The maltotriose binding site of MalT

The first conclusion of this work is that maltotriose binds in the large inner cavity of the sensor domain of MalT. The primary maltotriose binding site defined by the mutational analysis extends between the two main openings of the cavity and comprise residues from two pairs of head-to-tail stacked helix bundles: 2 and 7 on one hand, 3 and 8 on the other hand. Interestingly, the binding-site lies in the upper moiety of the pocket. This is unexpected because a glycerol molecule was present in the bottom of the pocket in the crystals used to solve the structure of the MalT sensor domain, leading to the notion that maltotriose might bind in that region (Steegborn et al., 2001). On the contrary, substitution of two arginines (R692 and R729) close to the glycerol in the crystal had virtually no effect on the affinity, and even increased transcription activation in vivo. One possible explanation for the latter observation is that these arginine residues are part of the binding site of a small molecule which would inhibit either maltotriose binding or subsequent signaling.

The primary maltotriose binding site shows classical features of carbohydrate binding sites.

Carbohydrate-binding sites have several characteristics that reflect the particular and amphiphilic nature of their ligands. Their most outstanding feature is the high frequency of two kinds of residues with different properties: aromatic residues that either stack against the pyranoside ring or establish Van der Waals contacts, and residues with polar planar groups that are suited to form 
1 bidentate hydrogen bonds, as commonly observed in protein carbohydrate complexes (Quiocho, 1989). In that respect, the proposed maltotriose binding site is fairly satisfactory, since all group 1 residues but one (F686) belong to an aminoacid type that is more frequent in the sugar-binding sites of the Procarb40 database (Malik and Ahmad, 2007) than in the whole protein sequences of the same database. Furthermore, the repartition of the different residues in the proposed binding site makes sense: F686 lies in a central position, while residues with planar polar groups at the periphery (E496, Q533, E536 H499, Q688, D726) are well positioned for an interaction with the equatorial hydroxyl groups of the glycosyl units; finally, at the expense of a local conformational change, W529 could stack against the hydrophobic side (A-face) of the glycosyl moiety at the non-reducing end of maltotriose. The fact that each one the two aromatic residues of the primary maltotriose-binding site is close to an opening of the pocket suggests that they might also be involved in the translocation of maltotriose through these openings to its final binding site. Indeed, stacking interactions are often involved in guided diffusion of polysaccharides (Divne et al., 1998; Schirmer et al., 1995). However, the proposed model (Fig. $4 \mathrm{~A}, \mathrm{C}$ ) is not necessarily exact in all its details: protein-carbohydrate complex structures generally show a denser network of hydrogen bonds (Quiocho, 1989). It must also be recalled that the alanine-scanning was restricted to residues conserved in a sample of MalT orthologs, so that a nonconserved secondary binding determinant could have been missed.

Finally, the shape of the primary maltotriose binding site proposed here provides a nice explanation for the specificity of MalT for maltotriose (Raibaud and Richet, 1987, see also Dippel et al., 2005). Maltodextrins longer than 3 glucosyl units are too long to fit in, as shown by the poor effect of maltotetraose on the DT3 fluorescence spectrum (Fig. 3B). On the other hand, the binding site provides interacting partners all along the maltotriose molecule, which would explain the preference for maltotriose over maltose (Fig. 3B and Table 2). Interestingly, the specificity for maltotriose over maltose seems stronger in the whole protein (Raibaud and Richet, 1987) than in the isolated sensor domain. It is therefore likely that, in addition to its lower affinity for the sensor domain, maltose is also less efficient in triggering the conformational changes that ensue.

\section{Comparison with other tetratricopeptide domains}

From the beginning, TPR domains, the closest structural relatives of the MalT SUPR domain, have been recognized as protein-protein interaction domains. Typically, binding of their partner protein has little if any effect on their conformation (Grove et al., 2008). However, several TPR proteins have recently been shown to diverge from this simple model. In the case of protein phosphatase 5 , the 
1 ligand has been proposed to act allosterically on the TPR domain. Namely, binding of its Hsp90 Cterminal peptide ligand slightly changes the conformation of the protein phosphatase 5 TPR domain in

3 a way that probably alters its inhibitory interaction with the catalytic domain (Cliff et al., 2006). In

4 another system, recognition of the peroxisomal targeting signal PTS1 by the PEX5 peroxisomal receptor was also shown to involve a long distance conformational change of the TPR domain of PEX5 (Stanley et al., 2006). Interestingly, the PEX5 TPR domain has the shape of a flattened cylinder with a central channel in which the PTS1 peptide binds, resulting in constriction of the channel due to a relative movement of the $\mathrm{C}$-terminal helix bundles with respect to the $\mathrm{N}$-terminal ones.

In the case of MalT, several observations point towards a maltotriose-induced conformational change of the SUPR domain. Assuming that maltotriose first binds to the primary maltotriose bindingsite presented here, the involvement of R574 in the binding of the inducer could suggest a contraction of the structure analogous to that observed in PEX5, which would shrink the cavity and enable this residue to interact with maltotriose.

\section{Maltotriose binding and signal transduction}

As said above, the $\beta$-galactosidase activities presented here are the outcome of a signal transduction process, whereas the fluorimetry experiments probe only one step of the signaling: maltotriose binding by the isolated sensor domain. Nevertheless, the two kinds of experiments give a relatively good correlation (Table 4). For instance, all 13 mutations causing more than a 10-fold reduction in maltotriose affinity for the sensor domain decrease the $\beta$-galactosidase activity of pop8083 by at least 3-fold and 5 out of the 11 mutations causing less than a 3-fold effect on maltotriose affinity for the sensor domain confer a $\beta$-galactosidase activity superior or equal to that of the wt allele. However the correlation is broken for the 6 remaining mutations that only mildly affect maltotriose affinity. I537A gives a relatively low $\beta$-galactosidase level (46\%), for which there is no obvious explanation. As to the remaining substitutions which barely alter the affinity of the sensor domain for maltotriose but confer a very low (12-25\%) $\beta$-galactosidase activity (N461A, E570A, F571A, L600A, W648A), they are involved, not in maltotriose binding but in another step of the signal transduction process. The fact that they cluster around an opening of the cavity (Fig. 4B) further suggests that this step consists in establishing or destroying a contact between these residues and another part of the protein (for instance the NOD-arm region). How such an interaction between the sensor and the NODarm region enables maltotriose to regulate the NOD switch and whether a similar scheme also applies 
1 to other STAND proteins with sensor domains of the SUPR and tetratricopeptide family remains to be 2 determined.

4 Experimental procedures

\section{Strains and plasmids}

pop8080 is derived from pop3125 (MC4100 (ФmalPQ-lacZY), Débarbouillé et al., 1978) as follows: an $\mathrm{F}$ factor has been introduced and the joint of the malPQ-lacZY fusion has been replaced by that of the malP'-'lacZ' translational fusion of phage M13malP3 (Danot and Raibaud, 1993), using transfer from M13 onto the chromosome as described by Danot and Raibaud (1993). pop8081 differs from pop8080 by a deletion encompassing the entire malT gene which was transferred from M13mp11::malTAmalT220 (Schreiber et al., 2000) onto the chromosome. pop8083 and pop8084 were built from pop8080 and pop8081, respectively, by replacing the malK-lamB-malM operon by a cassette conferring zeocin resistance (Richet, unpublished results).

M13malT9 was obtained by cloning the malT Hpal-Acc65I fragment of pOM180 (Richet et al., 2005) that encompasses the malT fragment encoding the sensor domain (DT3), into the Xbal site of M13mp11 DNA after filling in all cohesive ends, the 'ma/T' and 'lac' ORFs being divergent. M13malT9 4 lac is derived from M13malT9 by a 420 bp deletion starting immediately downstream of the EcoRI site of the polylinker and removing the whole 'lac' sequence.

All the mutations except one (Table S1) were introduced in M13malT9 by mutagenesis by the method of Kunkel (1987). To facilitate transfer to the chromosome in the case of W648A, Q690A, N693A and R729A, the BssHII-BssHII fragment of the M13malT9 derivatives carrying these mutations were cloned between the BssHII sites of M13malT9Llac. All the malT sequences in these phages were checked. Mutations were then transferred from M13malT9 or M13malT94lac onto the chromosomal malT gene of pop8080 as described (Danot and Raibaud, 1993). The success of the transfer was verified by amplifying the DT3 encoding fragment and digesting the amplicon by the relevant restriction enzyme (see Table S1) or by sequencing it in the case of R729A. Finally, all the pop8080 derivatives were converted into pop8083 derivatives (i.e. malK lamB- MalM) by P1 transduction using a P1 lysate of pop8083 followed by zeocin selection.

pOM164 and derivatives were constructed as follows: pET28b(+) (Novagen) was first rid of its unique BssHII site by amplifying with Pwo DNA polymerase (Roche) the whole plasmid DNA with oligonucleotides ET1 (creating a silent mutation in the lacl BssHII site; 
1 5'-IGCCATTACCGAGTCCGGGCT) and ET2 (5'-CGCATTGCGCCCAGCGCCA). The Ncol-HindIII

2 fragment of pOM152 that encodes DT3 was inserted into the same sites of this $\mathrm{pET} 28 \mathrm{~b}(+)$ derivative,

3 to give pOM164. pOM164H is derived from pOM164 by inserting the HTAG1-HTAG2 double stranded

4 oligonucleotide (Danot, 2001) in the Ncol site. The malT mutations were transferred into pOM164 by

5 cloning the small BssHII-BssHII fragment of the mutagenized M13malT9 into the BssHII sites of pOM164, except for mutations $N 693 A$ and $L 723 A$, which create a new BssHII site: in this case, the fragment of M13malT9 that encodes DT3 was PCR amplified using oligonucleotides DT3G and DT3D (Danot, 2001), digested by $\mathrm{Ncol}$ and HindlII and cloned between the same sites of pOM164. pOM164HR692A was constructed by cloning the small BssHII-BssHII fragment of M13malT9R692A into the BssHII sites of $\mathrm{pOM} 164 \mathrm{H}$ as a receiver.

$\beta$-galactosidase assay

Cells were grown in M9 medium (Sambrook et al., 1989) supplemented with $0.4 \%$ sorbitol. Cultures were inoculated with $100 \mu \mathrm{l}$ overnight precultures and grown at $37^{\circ} \mathrm{C}$, or inoculated with $1-10$ $\mu \mathrm{l}$ of a day preculture and grown overnight at $25^{\circ} \mathrm{C}$. When they reached an $A_{600}$ of $0.5-0.8$, $\beta$-galactosidase was assayed as described (Miller, 1972), using chloroform and $0.0019 \%$ SDS to permeabilize the cells and using DTT ( $0.5 \mathrm{mM}$ final concentration) instead of 2-mercaptoethanol.

\section{Western blots}

Cells were grown at $25^{\circ} \mathrm{C}$ as for the $\beta$-galactosidase assays. For each strain, cells (the equivalent of $5 \mathrm{ml}$ at $\left.\mathrm{A}_{600}=0.5\right)$ were collected and resuspended in $500 \mu \mathrm{l}$ of $50 \mathrm{mM}$ Tris- $\mathrm{HCl}(\mathrm{pH} 7.7), 10 \%$ sucrose, $0.3 \mathrm{M} \mathrm{KCl}, 1 \mathrm{mM}$ ATP. Cells were disrupted by sonication, and centrifuged in a labtop centrifuge (Eppendorf) at $13000 \mathrm{rpm}$ for $60 \mathrm{~min}$. Soluble extracts $(5 \mu \mathrm{l})$ were loaded on an SDS-PAGE gel containing $9.3 \%$ acrylamide and $0.12 \%$ bisacrylamide. Proteins were transferred on a Hybond ${ }^{\mathrm{TM}} \mathrm{C}$ membrane, probed with anti-MalT antibodies and a horse-radish peroxidase coupled secondary antibody. As a control, membranes were stripped and reprobed with an anti-sigma ${ }^{70}$ antibody. Membranes were treated with the ECL+ reagents (GE Healthcare, Fairfield, CT) and scanned on a Storm 840 (GE Healthcare).

\section{Protein purification}

$\mathrm{BL21(DE3)} \triangle$ malT220 carrying pOM164 or derivatives thereof were grown at $37^{\circ} \mathrm{C}$ in $200 \mathrm{ml} \mathrm{LB}$ 
1 medium $+50 \mu \mathrm{g} / \mathrm{ml}$ kanamycine to $A_{600}=2$ and induced with $0.1 \mathrm{mM}$ IPTG. Growth was continued at $224^{\circ} \mathrm{C}$ and cells were harvested $5 \mathrm{~h}$ later, washed and resuspended in $5 \mathrm{ml} \mathrm{TSK}$ buffer $(50 \mathrm{mM}$ Tris- $\mathrm{HCl}$ 3 (pH 7.7), 10\% sucrose, 0.5M KCl). In the case of hDT3 (His-tagged DT3), hR692A, E570A, N693A and 4 D726A, $200 \mathrm{ml}$ ZYP5052 medium (Studier, 2005) without trace metals and with bactotryptone instead 5 of $\mathrm{N}-\mathrm{Z}$ amine $+100 \mu \mathrm{g} / \mathrm{ml}$ kanamycine were inoculated at $\mathrm{A}_{600}=0.01$ with BL21(DE3) $\Delta$ malT220 carrying pOM164H, pOM164HR692A, pOM164E570A, N693A and D726A respectively. Bacteria were grown under strong agitation $(200 \mathrm{rpm})$, at $16^{\circ} \mathrm{C}$ for $48 \mathrm{~h}(\mathrm{hDT} 3, \mathrm{hR692 \textrm {A }})$ or at $22^{\circ} \mathrm{C}$ for $22-28 \mathrm{~h}$ (N693A, D726A, E570A) washed and resuspended in $12 \mathrm{ml} \mathrm{TSK} \mathrm{buffer.}$

Cells were disrupted with a French press at 16000 psi after addition of $20 \mu \mathrm{g} / \mathrm{ml}$ DNasel and

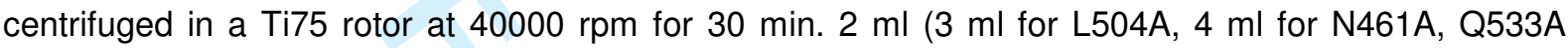
E570A and D726A, $5 \mathrm{ml}$ for E496A, $14 \mathrm{ml}$ for hR692A) of the supernatant were loaded on a $1 \mathrm{ml} \mathrm{Ni}$ NTA (Qiagen) column. The column was washed with TSK buffer, TSK buffer $+5 \mathrm{mM}$ imidazole and eluted in TSK buffer + $20 \mathrm{mM}$ imidazole, except for hR692A and hDT3, for which the columns were washed with TSK buffer, TSK buffer $+40 \mathrm{mM}$ imidazole and eluted with TSK $+400 \mathrm{mM}$ imidazole. Proteins were recovered at concentrations (determined by the method of Lowry (1951)) between 5 and $15 \mathrm{mg} / \mathrm{ml}$ except for hDT3 $(30 \mathrm{mg} / \mathrm{ml}$ ) and their purity was over $80 \%$ (as estimated by quantification of Coomassie Blue stained gels loaded with different protein amounts) except for E496A and hR692A 18 (75\%).

Fluorimetry

Fluorescence measurements were performed on a PTI (Lawrenceville, NJ, USA) QuantaMaster ${ }^{\mathrm{TM}}$ (or PTI QuantaMaster QM4CW for the experiments of Fig. 3B) spectrofluorometer at $20^{\circ} \mathrm{C}$ on $1 \mathrm{ml}$ samples containing $1 \mu \mathrm{M}$ protein in a $50 \mathrm{mM}$ Tris- $\mathrm{HCl}(\mathrm{pH} 7.7), 0.1 \mathrm{M} \mathrm{KCl}$ degassed buffer under continuous stirring. The excitation wavelength was $284 \mathrm{~nm}$, excitation and emission bandwidths were 1 and $4 \mathrm{~nm}$, respectively. Fluorescence emission spectra were recorded between 300 and $450 \mathrm{~nm}$ in the absence and in the presence of the indicated maltotriose concentration. The spectral center of gravity was calculated as:

$$
\frac{\sum_{\lambda=300}^{420} \lambda F(\lambda)}{\sum_{\lambda=300}^{420} F(\lambda)}
$$

with $\lambda$ designating the wavelength in $\mathrm{nm}$ and $F(\lambda)$ the fluorescence intensity in arbitrary units at 
1 that wavelength. Maltotriose binding studies were carried out by adding increasing concentrations of maltotriose to the sample without exceeding a $5 \%$ dilution and by recording fluorescence emission once per second at the indicated wavelength (between 316 and $338 \mathrm{~nm}$ ) during $100 \mathrm{~s}$ after each maltotriose addition. Each point is the average of 50 scans, generally those between $46 \mathrm{~s}$ and $95 \mathrm{~s}$. In a control experiment using DT3, two spectra were recorded instead of the 100 scans after each maltotriose addition. The $K_{D}$ which were calculated for each wavelength between 315 and $350 \mathrm{~nm}$ were all within $10 \%$ of $3.3 \mathrm{mM}$ (not shown), suggesting that the wavelength used does not affect importantly the calculation of the dissociation constant.

The overall shape of the DT3 fluorescence spectrum repeatedly obtained in this work with the PTI QuantaMaster was different from that of Danot (2001) (Fig. 3A). The new spectrum showed a "flat top", with fluorescence emission intensity only slowly increasing between 342 and $348 \mathrm{~nm}$, where the maximum is attained. This spectrum was reproducibly obtained for several years with different DT3 preparations, and it undergoes similar changes upon maltotriose addition as that of Danot (2001) (a 12 $\%$ increase in the maximum fluorescence and a blue-shift of $1.7 \pm 0.32 \mathrm{~nm}$ of the spectral center of gravity, with a new $K_{D}$ measurement of $3.5 \mathrm{mM}$ ). A control experiment was carried out on a Perkin Elmer LS 50B spectrofluorometer: the Perkin Elmer spectrum obtained had the same overall shape as the new spectrum (with a shift to longer wavelengths, which might reflect differences in the calibration and in the optics of the two spectrofluorometers), and the effect of maltotriose was similar (Fig. S1; shift in the spectral center of gravity: $1.7 \mathrm{~nm}$ ). Finally, the shape of the spectrum obtained with the recently purchased PTI QuantaMaster QM4CW (Fig. 3B, $\mathrm{K}_{\mathrm{D}}$ measured for maltotriose, $2.7 \mathrm{mM}$, increase in maximum fluorescence $12 \%$, shift in the spectral center of gravity: $1.6 \mathrm{~nm}$ ), was much closer to that of Danot (2001), suggesting that ageing of components of the PTI Quantamaster was the cause of the difference observed between Fig. 3A and Danot (2001).

\section{Automated docking}

Automated docking was performed using Autodock 4.0 (Goodsell and Olson, 1990; Morris et al., 1998; Morris et al., 2009). Energy grids of $63 \times 87 \times 68$ points centered on the $(-25.76 ; 55.38 ;-42.963)$ point with a spacing of $0.375 \AA$ were calculated using the $1 \mathrm{hz} 4$ structure of the Protein Data Bank. Grids contained all atoms of residues from group 1-3. Preparation of the ligand and protein files was performed using the AutodockTools 1.4 .5 package. Nine runs of 50 dockings of the maltotriose molecule (idealized coordinates from the MSDchem database (http://www.ebi.ac.uk/msdsrv/msdchem)) were performed using the Lamarckian genetic algorithm with default parameters 
1 except for the population size (300) and number of energy evaluations $\left(10^{8}\right)$. The average distance

2 from a ligand pose to group 1 residues was measured as the mean over all ligand atoms of the 3 minimum over all group 1 residue atoms of the distances between the ligand atoms and group 1

4 residue atoms.

5

Limited proteolysis and microsequencing Eight $\mu \mathrm{g}$ DT3 or GST-'T (Vidal-Ingigliardi et al., 1993) were preincubated for $5 \mathrm{~min}$ at $25^{\circ} \mathrm{C}$ in $26 \mu \mathrm{l}$ of $62 \mathrm{mM}$ Tris- $\mathrm{HCl}(\mathrm{pH} 7.7), 0.38 \mathrm{M} \mathrm{KCl}, 0.8 \%$ sucrose $(0.9 \%$ glycerol for GST-'T) and 0, $200 \mathrm{mM}$ maltotriose or $200 \mathrm{mM}$ glucose. Reactions were initiated by addition of $4 \mu \mathrm{l}$ trypsin at different concentrations and stopped after 30 min by adding $10 \mu$ AEBSF (4(2-aminoethyl) benzenesulfonyl fluorode hydrochloride) at $16 \mathrm{mM}$ and $40 \mu \mathrm{l}$ of sample buffer $(0.125 \mathrm{M}$ Tris- $\mathrm{HCl} \mathrm{pH}$ (6.8), $4 \%$ SDS, $20 \%$ glycerol, $0.01 \%$ bromophenol blue). Ten $\mu \mathrm{l}$ were loaded on a $12 \%$ (37.5:1 acrylamide-bisacrylamide) SDS-PAGE. The gel (0.8 mm thickness) was stained using Sypro® Ruby (BioRad, Hercules, CA) $\left(0.1 \mathrm{ml} / \mathrm{cm}^{2}\right)$ according to the manufacturer specifications and scanned with a STORM 840 (GE Healthcare).

For $\mathrm{N}$-terminal microsequencing of the H645A tryptic fragment, $12 \mu \mathrm{g} \mathrm{H645A} \mathrm{were} \mathrm{digested} \mathrm{as}$ above without maltotriose in $20 \mu \mathrm{l}$ at $35 \mu \mathrm{g} / \mathrm{ml}$ trypsin. Digested products were transferred to a Hybond $^{\mathrm{TM}} \mathrm{P}$ membrane (GE Healthcare) and microsequenced at the Microsequencing Platform of the

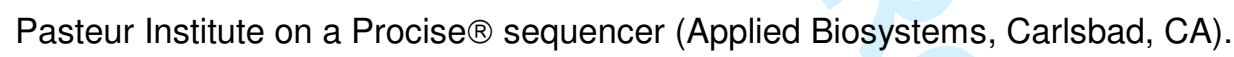

\section{Acknowledgements}

The author thanks E. Richet for many stimulating discussions, for her comments on the manuscript and for the gift of purified GST-'T and of the zeocin resistance cassette, C. Steegborn for the gift of plasmid pOM152-R729A, Dominique Vidal-Ingigliardi for the gift of M13 DNA carrying the $\Delta / a c$ deletion, F. Agou, B. Baron, J.-M. Betton, A. Chaffotte and P. England for their advice and help with fluorimetry, $\operatorname{Dr}$ A. J. Olson for providing the Autodock 4.0 software, and T. Pugsley for his interest in this work. This work was funded by the Agence Nationale de la Recherche (ANR-08-BLAN-0204-01). 


\section{TABLES}

\begin{tabular}{ccc} 
& \multicolumn{2}{c}{ Spectral center of gravity $(\mathrm{nm})$} \\
\cline { 2 - 3 } MalT variant & - maltotriose & + maltotriose \\
\hline DT3 & $349.9 \pm 0.17$ & $348.2 \pm 0.15$ \\
E570A & $349.7 \pm 0.18$ & $349.1 \pm 0.07$ \\
D647A & $349.6 \pm 0.21$ & $349.2 \pm 0.12$ \\
W648A & $348.8 \pm 0.09$ & $348.1 \pm 0.16$ \\
D726A & $348.6 \pm 0.28$ & $348.3 \pm 0.27$ \\
\hline
\end{tabular}

Table 1: Effect of particular substitutions on the maltotriose induced blue-shift. Spectral centers of

3 gravities were measured between 300 and $420 \mathrm{~nm}$ for particular mutants as indicated in 4 Experimental Procedures. Each value is the mean of at least 4 independent experiments. 
1

\begin{tabular}{lcl} 
MalT variant & \multicolumn{1}{c}{$\mathrm{K}_{\mathrm{D}}(\mathrm{mM})$} \\
\hline DT3 & $3.5 \pm 0.6 \quad(325)$ \\
hDT3 & $4.6 \pm 0.6 \quad(325)$ \\
Q457A & $22 \pm 7$ & $(317,321)$ \\
N461A & $5.1 \pm 0.3 \quad(321)$ \\
L504A & $46 \pm 19.5 \quad(316)$ \\
I537A & $4.6 \pm 0.5$ & $(321,325)$ \\
E570A & $4.4 \pm 0.5$ & $(321,334)$ \\
F571A & $5.7 \pm 0.8$ & $(328,334)$ \\
L600A & $8.3 \pm 0.5$ & $(321)$ \\
D647A & $9.2 \pm 0.8$ & $(321,332)$ \\
W648A & $6.0 \pm 0.6$ & $(321)$ \\
H685A & $20 \pm 4$ & $(321)$ \\
Q688 & $83 \pm 48$ & $(316)$ \\
Q690A & $2.7 \pm 0.3$ & $(325,332)$ \\
hR692A & $13 \pm 2$ & $(321)$ \\
N693A & $6.8 \pm 0.3 \quad(323,329)$ \\
L723A & $45 \pm 18$ & $(321)$ \\
R729A & $5.4 \pm 1.3 \quad(321,338)$ \\
\hline
\end{tabular}

2

3 Table 2: Dissociation constants of MalT variants for maltotriose measured by fluorimetry.

4 Each value is the average of two independent experiments, except for DT3 (four experiments).

5 Number in parentheses give the emission wavelength(s) used for each experiment. The dissociation

6 constant of DT3 and maltose measured in the same conditions was $28.6 \pm 8.8 \mathrm{mM}$. 
1

cluster $\mathrm{n}^{\circ} \quad$ energy $(\mathrm{kcal} / \mathrm{mol}) \quad$ population distances to group1

\begin{tabular}{rrrc} 
& & & average (max-min) in $\AA$ \\
\hline 1 & -13.6 & 3 & $3.87(3.69-4.17)$ \\
5 & -12.3 & 18 & $4.25(3.79-4.38)$ \\
7 & -12.1 & 24 & $4.04(4.01-5.52)$ \\
15 & -12.0 & 18 & $3.52(3.34-3.74)$ \\
\hline
\end{tabular}

2 Table 3: Summary of the automated dockings. Data concerning the lowest energy cluster and the 4

3 most populated clusters are presented. The last column gives the average, maximum and minimum

4 over all conformations of the cluster with an energy $<-11.1 \mathrm{kcal} / \mathrm{mol}$ of the average distance

5 between the ligand and group 1 residues (as defined in Experimental procedures). 
1

\begin{tabular}{|c|c|c|c|}
\hline MalT variant & $\begin{array}{l}\text { numbering of } \\
\text { PDB file }\end{array}$ & $\mathrm{K}_{\mathrm{D}} / \mathrm{K}_{\mathrm{D}}(\mathrm{wt})$ & $\begin{array}{c}\beta \text {-galactosidase activity } \\
(\% \text { of } w t)\end{array}$ \\
\hline Q457A & 20 & 6.3 & 18 \\
\hline N461A & 24 & 1.5 & 12 \\
\hline E496A & 59 & - & 11 \\
\hline H499A & 62 & - & 20 \\
\hline L504A & 67 & 13.2 & 24 \\
\hline W529A & 92 & - & 6 \\
\hline Q533A & 96 & - & 14 \\
\hline E536A & 99 & - & 34 \\
\hline I537A & 100 & 1.3 & 46 \\
\hline E570A & 133 & 1.3 & 15 \\
\hline F571A & 134 & 1.6 & 18 \\
\hline R574A & 137 & - & 9 \\
\hline W581A & 144 & N.D. & 0 \\
\hline L600A & 163 & 2.4 & 18 \\
\hline $\mathrm{H} 645 \mathrm{~A}$ & 208 & - & 16 \\
\hline D647A & 210 & 2.6 & 101 \\
\hline W648A & 211 & 1.7 & 25 \\
\hline N684A & 247 & - & 26 \\
\hline H685A & 248 & 5.6 & 47 \\
\hline F686A & 249 & - & 33 \\
\hline Q688A & 251 & 23.7 & 14 \\
\hline Q690A & 253 & 0.8 & 116 \\
\hline R692A & 255 & $2.9^{\mathrm{a}}$ & 206 \\
\hline N693A & 256 & 2.0 & 122 \\
\hline R696A & 259 & N.D. & 1 \\
\hline L723A & 286 & 12.8 & 26 \\
\hline D726A & 289 & - & 11 \\
\hline R729A & 292 & 1.5 & 245 \\
\hline I769A & 332 & N.D. & 1 \\
\hline
\end{tabular}

2 Table 4: Summary of the results. The fluorescence data of the purified proteins and the $\beta$ 3 galactosidase activities of the pop8084 strains producing the different MalT variants are presented as

4 ratios to the data obtained with the wt. - means that the $K_{D}$ was too high to be measured. N.D.

5 indicates that the protein could not be purified. 
$1{ }^{a}$ Ratio between the $K_{D}$ of $h R 692$ and that of $h D T 3$. 
2 Figure legends

4 Fig. 1. (A) Representation of the MalT primary sequence with indication of the boundaries of the domains. AAA+: conserved core of AAA+ ATPases. The MalT effector domain is a DNA-binding domain. (B) Schematic representation of the MalT sensor domain. The aminoacid sequence is shown with boxes representing the helices arranged in bundles as in the three-dimensional structure. Numbering starts with the first residue of the MalT protein, with arrowheads every ten residues. Residues substituted in this work are circled. Inset: three dimensional representation from $1 \mathrm{hz} 4$ entry of the Protein Data Bank (http://www.rcsb.org) with cylindrical helices, numbered from the beginning of the domain. Bundle 1 is made of helices 1 and 2, and so on. Even and odd helices are colored in light grey and black, respectively, to show the two-layer nature of the superhelix. The three helices that are not numbered compose the $\mathrm{C}$-terminal subdomain.

(C) Surface representation of the MalT sensor domain with residues of the mouths of the central cavity (as defined by CASTp) represented in blue (axial mouth) and cyan (lateral mouth). Left: view along the axis of the cavity. Center: front view, as in the inset of Fig.1B. Right: left view. Rotation angles and axes (going from the left to the right panel) are indicated.

Fig. 2. Effect of the mutations on transcription activation by MalT in vivo. $\beta$-galactosidase activities of cultures of pop8083 and its mutated derivatives grown at $25^{\circ} \mathrm{C}$ were calculated as the mean of at least three independent experiments. A background of 52 Miller units was subtracted from all the activities. Error bars correspond to one standard deviation. Bottom panel: western blot of soluble extracts of the same strains grown in the conditions of the $\beta$-galactosidase assays, revealed by anti-MalT and antisigma $^{70}$ antibodies as indicated. The three right hand panels are croppings of the scan of one membrane while the left hand panel is a scan of another membrane.

Fig. 3. Fluorescence spectra and binding curves. (A) Fluorescence emission spectra recorded at $20^{\circ} \mathrm{C}$ after excitation at $284 \mathrm{~nm}$ without (blue) and with maltotriose (red) are shown, reassembled according to the group they belong to (see text). The group is indicated in the upper left corner of the panel.

30 Spectra without maltotriose have been normalized to have the same maximal value as the wt, and for each variant the spectrum with maltotriose was normalized by the same factor. Each pair of spectra is 
1 vertically shifted from the precedent pair for clarity. A.U. : fluorescence arbitrary units. The buffer spectrum of the wt experiment is drawn in black. Maltotriose concentrations used for the spectra with maltotriose were: Q690A, R729A: 15 mM; E570A, F571A, W648A: 20mM; wt, N461A, I537A, L600A, D647A, H685A: 25 mM; N693A, hR692A, L723A, 30 mM; Q457A, L504A : 40 mM; E496A, H499A, W529A, Q533A, E536A, R574A, N684A, H645A, F686A, Q688A, D726A: 50 mM. Note that saturation by maltotriose could not always be obtained. Percentages of saturation calculated from the binding curve fits are the following: wt: 86\%; Q457A: 61\%; N461A: 81\%; L504A: 65\%; I537A: 81\%; E570A: 80\%; F571A: 78\%; L600A: 74\%; D647A: 68\%; W648A: 74\%; H685A: 55\%; Q688A: 38\%; Q690A: 82\%; hR692A: 69\%; N693A: 81\%; L723A: 38\%; R729A: 74\%. (B) Fluorescence emission spectra of wt DT3 $\left(20^{\circ} \mathrm{C}\right.$, excitation at $\left.284 \mathrm{~nm}\right)$ in the presence of $25 \mathrm{mM}$ maltotriose, maltose or maltotetraose. The difference in the wt spectra between Fig.3A and 3B is due to the use of two different fluorometers (see Experimental Procedures). (C) Examples of binding curves. See Experimental Procedures and Fig. S2 for details.

Fig. 4. Structure-function relationship in DT3. (A) Surface representation of the MalT sensor domain with a docked maltotriose molecule (see text) split in two to reveal the inner wall of the pocket. The left moiety (same viewing axis as in Fig. 1C (center panel)) contains most of bundles 1, 2, 3, 6, 7 and 8 while the right comprises most of bundles 4 and 5 and the 3-helix subdomain. Color code: group 1 residues: red; group 2, orange; group 3, yellow; group 4, green; maltotriose, cyan.. The axial opening is indicated by a vertical arrow, the lateral one by a horizontal arrow. (B) Top view. The rotation indicated transforms the viewing axis of (A, left panel) in that of (B). Residues whose alanine substitution does not affect the $K_{D}$ but decreases the $\beta$-galactosidase activity of pop8083 are indicated. Same color code as in (A). (C) Stereo view of a sticks representation of the primary maltotriose binding-site with docked maltotriose in the conformation shown in (A). All group 1-3 residues are shown, with hypothetical hydrogen bonds in green dashed lines. R692 is also represented even though it belongs to group 4 because it is predicted to donate a hydrogen bond to the maltotriose in this conformation. Backbone, R692 and maltotriose atoms are colored according to their nature (C: grey (backbone, R692) or cyan (maltotriose), $\mathrm{O}$, red, $\mathrm{H}$, white). Side-chains are colored according to the color-code of (A).

Fig. 5. Limited proteolysis of the MalT sensor domain in the presence and absence of maltotriose or glucose. + indicates the presence of $200 \mathrm{mM}$ of maltotriose or glucose. Lanes $0-14$, DT3; trypsin concentrations in $\mu \mathrm{g} / \mathrm{ml}: 0$ (lane 0), 2.1 (lane 1), 4.2 (lanes 2, 6), 8.3 (lanes 3, 7), 17 (lanes 4, 8), 33 
1 (lanes 5, 9, 12 and 13), 67 (lane 10 and 14). Lanes 15-17, GST-'T; lane 15, undigested; lanes 16,17,

$21.9 \mu \mathrm{g} / \mathrm{ml}$ trypsin. Samples in lanes $15-17$ have been run on the same gel.

3

4

5

6

7

8

Ade, J., DeYoung, B.J., Golstein, C., and Innes, R.W. (2007) Indirect activation of a plant nucleotide binding site-leucine-rich repeat protein by a bacterial protease. Proc. Natl. Acad. Sci. U S A 104: 2531-2536.

Bao, Q., Lu, W., Rabinowitz, J.D., and Shi, Y. (2007) Calcium blocks formation of apoptosome by preventing nucleotide exchange in APAF-1. Mol. Cell 25: 181-192.

Bikadi, Z., and Hazai, E. (2009) Application of the PM6 semi-empirical method to modeling proteins enhances docking accuracy of AutoDock. J. Cheminform. 1: 15.

Boos, W., and Shuman, H. (1998) Maltose/maltodextrin system of Escherichia coli: transport, metabolism, and regulation. Microbiol. Mol. Biol. Rev. 62: 204-229.

Cliff, M.J., Harris, R., Barford, D., Ladbury, J.E., and Williams, M.A. (2006) Conformational diversity in the TPR domain-mediated interaction of protein phosphatase 5 with Hsp90. Structure 14: 415426.

Danot, O., and Raibaud, O. (1993) On the puzzling arrangement of the asymmetric MalT-binding sites in the MalT-dependent promoters. Proc. Natl. Acad. Sci. USA 90: 10999-11003.

Danot, O. (2001) A complex signaling module governs the activity of MalT, the prototype of an emerging transactivator family. Proc. Natl. Acad. Sci. USA 98: 435-440.

Danot, O., Marquenet, E., Vidal-Ingigliardi, D., and Richet, E. (2009) Wheel of life, wheel of death: a mechanistic insight into signaling by STAND proteins. Structure 17: 172-182.

Débarbouillé, M., Shuman, H.A., Silhavy, T.J., and Schwartz, M. (1978) Dominant constitutive mutations in malT, the positive regulator gene of the maltose regulon in Escherichia coli. J. Mol. Biol. 124: 359-371.

Dippel, R., Bergmiller, T., Boehm, A., and Boos, W. (2005) The maltodextrin system of Escherichia coli: glycogen-derived endogenous induction and osmoregulation. J. Bacteriol. 187: 83328339. 
1 Divne, C., Stahlberg, J., Teeri, T.T., and Jones, T.A. (1998) High-resolution crystal structures reveal how a cellulose chain is bound in the $50 \AA$ long tunnel of cellobiohydrolase I from Trichoderma reesei. J. Mol. Biol. 275: 309-325.

Dundas, J., Ouyang, Z., Tseng, J., Binkowski, A., Turpaz, Y., and Liang, J. (2006) CASTp: computed atlas of surface topography of proteins with structural and topographical mapping of functionally annotated residues. Nucleic Acids Res. 34: W116-118.

Erzberger, J.P., Mott, M.L., and Berger, J.M. (2006) Structural basis for ATP-dependent DnaA assembly and replication-origin remodeling. Nat. Struct. Mol. Biol. 13: 676-683.

Goodsell, D.S., and Olson, A.J. (1990) Automated docking of substrates to proteins by simulated annealing. Proteins 8: 195-202.

Grove, T.Z., Cortajarena, A.L., and Regan, L. (2008) Ligand binding by repeat proteins: natural and designed. Curr. Opin. Struct. Biol. 18: 507-515.

Hsu, J.L., Peng, H.L., and Chang, H.Y. (2008) The ATP-binding motif in AcoK is required for regulation of acetoin catabolism in Klebsiella pneumoniae CG43. Biochem. Biophys. Res. Commun. 376: 121-127.

Hu, Y., Ding, L., Spencer, D.M., and Nuñez, G. (1998) WD-40 repeat region regulates APAF-1 selfassociation and procaspase-9 activation. J. Biol. Chem. 273: 33489-33494.

Huey, R., Morris, G.M., Olson, A.J., and Goodsell, D.S. (2007) A semiempirical free energy force field with charge-based desolvation. J. Comput. Chem. 28: 1145-1152.

Jia, Y., McAdams, S.A., Bryan, G.T., Hershey, H.P., and Valent, B. (2000) Direct interaction of resistance gene and avirulence gene products confers rice blast resistance. EMBO J. 19: 4004-4014.

Kumar, P., Kumar, D., Parikh, A., Rananaware, D., Gupta, M., Singh, Y., and Nandicoori, V.K. (2009) The Mycobacterium tuberculosis protein kinase $\mathrm{K}$ modulates activation of transcription from the promoter of mycobacterial monooxygenase operon through phosphorylation of the transcriptional regulator VirS. J. Biol. Chem. 284: 11090-11099.

Kunkel, T.A., Roberts, J.D., and Zakour, R.A. (1987) Rapid and efficient site-specific mutagenesis without phenotypic selection. Methods in Enzymology 154: 367-382.

Leipe, D.D., Koonin, E.V., and Aravind, L. (2004) STAND, a class of P-loop NTPases including animal 
and plant regulators of programmed cell death: multiple, complex domain architectures, unusual phyletic patterns, and evolution by horizontal gene transfer. J. Mol. Biol. 343: 1-28.

Lowry, O.H., Rosebrough, N.J., Farr, A.L., and Randall, R.J. (1951) Protein measurement with the Folin phenol reagent. J. Biol. Chem. 193: 265-275.

Main, E.R., Xiong, Y., Cocco, M.J., D'Andrea, L., and Regan, L. (2003) Design of stable alpha-helical arrays from an idealized TPR motif. Structure 11: 497-508.

Malik, A., and Ahmad, S. (2007) Sequence and structural features of carbohydrate binding in proteins and assessment of predictability using a neural network. BMC Struct. Biol. 7: 1.

Marquenet, E., and Richet, E. (2007) How integration of positive and negative regulatory signals by a STAND signaling protein depends on ATP hydrolysis. Mol. Cell 28: 187-199.

Miller, J.H. (1972) Experiments in Molecular Genetics. Cold Spring Harbor Laboratory, Cold Spring Harbor, NY, USA.

Moffett, P., Farnham, G., Peart, J., and Baulcombe, D.C. (2002) Interaction between domains of a plant NBS-LRR protein in disease resistance-related cell death. EMBO J. 21: 4511-4519.

Morris, G.M., Goodsell, D.S., Huey, R., and Olson, A.J. (1996) Distributed automated docking of flexible ligands to proteins: parallel applications of AutoDock 2.4. J. Comput. Aided Mol. Des. 10: $293-304$.

Morris, G.M., Goodsell, D.S., Halliday, R.S., Huey, R., Hart, W.E., Belew, R.K., and Olson, A.J. (1998) Automated docking using a Lamarckian genetic algorithm and an empirical binding free energy function. J. Comput. Chem. 19: 1639-1662.

Morris, G.M., Huey, R., Lindstrom, W., Sanner, M.F., Belew, R.K., Goodsell, D.S., and Olson, A.J. (2009) AutoDock4 and AutoDockTools4: Automated docking with selective receptor flexibility. J. Comput. Chem.

Nayal, M., and Honig, B. (2006) On the nature of cavities on protein surfaces: application to the identification of drug-binding sites. Proteins 63: 892-906.

Quiocho, F.A. (1989) Protein-carbohydrate interactions: basic molecular features. Pure \& Appl. Chem. 61: 1293-1306.

Raibaud, O., and Richet, E. (1987) Maltotriose is the inducer of the maltose regulon of Escherichia coli. J. Bacteriol. 169: 3059-3061. 
1 Richet, E., Joly, N., and Danot, O. (2005) Two domains of MalT, the activator of the Escherichia coli maltose regulon, bear determinants essential for anti-activation by MalK. J. Mol. Biol. 347: 110.

Riedl, S.J., Li, W., Chao, Y., Schwarzenbacher, R., and Shi, Y. (2005) Structure of the apoptotic protease-activating factor 1 bound to ADP. Nature 434: 926-933.

Sambrook, J., Fritsch, E.F., and Maniatis, T. (1989) Molecular cloning. Cold Spring Harbor, N.Y.: Cold Spring Harbor Laboratory press.

Schirmer, T., Keller, T.A., Wang, Y.F., and Rosenbusch, J.P. (1995) Structural basis for sugar translocation through maltoporin channels at 3.1 A resolution. Science 267: 512-514.

Schreiber, V., and Richet, E. (1999) Self-association of the Escherichia coli transcription activator MalT in the presence of maltotriose and ATP. J. Biol. Chem. 274: 33220-33226.

Schreiber, V., Steegborn, C., Clausen, T., Boos, W., and Richet, E. (2000) A new mechanism for the control of a prokaryotic transcriptional regulator: antagonistic binding of positive and negative effectors. Mol. Microbiol. 35: 765-776.

Stanley, W.A., Filipp, F.V., Kursula, P., Schuller, N., Erdmann, R., Schliebs, W., Sattler, M., and Wilmanns, M. (2006) Recognition of a functional peroxisome type 1 target by the dynamic import receptor Pex5p. Mol. Cell 24: 653-663.

Steegborn, C., Danot, O., Huber, R., and Clausen, T. (2001) Crystal structure of transcription factor MalT domain III: a novel helix repeat fold implicated in regulated oligomerization. Structure 9: 1051-1060.

Studier, F.W. (2005) Protein production by auto-induction in high density shaking cultures. Protein Expr. Purif. 41: 207-234.

Tameling, W.I., Vossen, J.H., Albrecht, M., Lengauer, T., Berden, J.A., Haring, M.A., Cornelissen, B.J., and Takken, F.L. (2006) Mutations in the NB-ARC domain of I-2 that impair ATP hydrolysis cause autoactivation. Plant Physiol. 140: 1233-1245.

Tanabe, T., Chamaillard, M., Ogura, Y., Zhu, L., Qiu, S., Masumoto, J., Ghosh, P., Moran, A., Predergast, M.M., Tromp, G., Williams, C.J., Inohara, N., and Nuñez, G. (2004) Regulatory regions and critical residues of NOD2 involved in muramyl dipeptide recognition. EMBO J. 23: 1587-1597. 
1 Tanaka, A., Takano, Y., Ohnishi, Y., and Horinouchi, S. (2007) AfsR recruits RNA polymerase to the 2 afsS promoter: a model for transcriptional activation by SARPs. J Mol Biol 369: 322-333.

3 Vidal-Ingigliardi, D., Richet, E., Danot, O., and Raibaud, O. (1993) A small C-terminal region of the 4 Escherichia coli MalT protein contains the DNA-binding domain. J. Biol. Chem. 268: 245275 24530.

6 Zhao, Y.H., Abraham, M.H., and Zissimos, A.M. (2003) Fast calculation of van der Waals volume as a 7 sum of atomic and bond contributions and its application to drug compounds. J. Org. Chem. 8 68: 7368-7373. 